\title{
Intrathecal injection of phosphodiesterase 4B-specific siRNA attenuates neuropathic pain in rats with $\mathrm{L5}$ spinal nerve ligation
}

\author{
QING JI, YAN DI, XIAOYUN HE, QINGZHEN LIU, JIAN LIU, WEIYAN LI and LIDONG ZHANG \\ Department of Anesthesiology, Jinling Hospital, School of Medicine, \\ Nanjing University, Nanjing, Jiangsu 210002, P.R. China
}

Received February 4, 2015; Accepted November 30, 2015

DOI: $10.3892 / \mathrm{mmr} .2015 .4713$

\begin{abstract}
Phosphodiesterase 4 (PDE4) is an adenosine cyclic 3,5-monophosphate-specific degradative enzyme, which is closely associated with the inflammatory response. Among its four subtypes (A-D), it remains unclear which one exerts suppressive effects on inflammation and reduces neuropathic pain. The present study aimed to examine the modulation of neuroinflammation by PDE4 subtypes in the spinal cord of a rat model of L5 spinal nerve ligation (SNL)-induced neuropathic pain. The expression levels of PDE4A-D were measured in the lumbar spinal cords of naïve rats. The rats were then divided into seven groups: The sham group (sham surgery + saline), the saline group (SNL + saline), the vehicle group (SNL + Lipofectamine ${ }^{\circledR}$ RNAiMAX), the mismatch small interfering (si)RNA group (SNL + mismatch siRNA), the PDE4A-siRNA group (SNL + PDE4A-siRNA), the PDE4B-siRNA group (SNL + PDE4B-siRNA) and the PDE4D-siRNA group (SNL + PDE4D-siRNA). In order to determine behavioral changes, mechanical withdrawal threshold (MWT) and thermal withdrawal latency (TWL) were recorded. The mRNA and protein expression levels of PDE4s were also detected. Furthermore, the association between behavioral changes and individual subtypes of PDE4 were studied following intrathecal administration of PDE4A, B and D-specific siRNA. The expression levels of protein kinases, including phosphorylated-extracellular signal-regulated kinases ( $p-E R K)$, and inflammatory cytokines were measured, in order to explore the underlying mechanisms. Subtypes A, B and D, but not C, were detected in the naïve rats. After SNL, both MWT and TWL were reduced. The mRNA and protein expression levels of PDE4A, B and D were significantly upregulated after 2, 4,
\end{abstract}

Correspondence to: Mr. Lidong Zhang, Department of Anesthesiology, Jinling Hospital, School of Medicine, Nanjing University, 305 East Zhongshan Road, Nanjing, Jiangsu 210002, P.R. China

E-mail: jzwrite@163.com

Key words: phosphodiesterase 4B, neuropathic pain, glial cell, inflammation
6 and 8 days of SNL. Subtype-specific siRNA significantly suppressed the elevated expression levels; however, only rats treated with PDE4B siRNA exhibited improved MWT and TWL. Further analysis of the PDE4B siRNA-treated rats demonstrated that 8 days after SNL, the intensity of p-ERK was reduced, the expression levels of CD11b and glial fibrillary acidic protein GFAP were reduced, as well as the expression levels of proinflammatory cytokines such as tumor necrosis factor- $\alpha$, interleukin (IL)-1 $\beta$ and IL-6. These results suggested that selective inhibition of PDE4B may relieve neuropathic pain, potentially via the suppression of glial activation and the release of cytokines in the spinal cord.

\section{Introduction}

Neuropathic pain, which is characterized by spontaneous pain, hyperalgesia and allodynia, is a major clinical problem that remains difficult to treat (1). At present, pain relief can only be achieved in $40-60 \%$ of patients; therefore, it is important to study the underlying mechanisms of pain, with the aim of eventually developing novel therapeutic drugs (2). Previous studies have suggested that glial cell-mediated neuroinflammation has an important role in neuropathic pain; however, exactly how glial cells are involved remains unclear (3-5).

Adenosine cyclic 3,5-monophosphate (cAMP) is an ubiquitous regulator of inflammation and is a key second messenger that influences glial activity $(6,7)$. It has previously been reported that increasing levels of cAMP via various methods may suppress the activation of glial cells (both microglia and astrocytes), decrease the production of proinflammatory mediators, including tumor necrosis factor (TNF)- $\alpha$, interleukin (IL)-1 $\beta$, IL-6, IL-12 and nitric oxide, and increase the expression of anti-inflammatory factor IL-10 (8-10).

Phosphodiesterases (PDEs), which are responsible for the degradation of $\mathrm{cAMP}$, have an important role regulating the intracellular levels of cAMP $(11,12)$. PDEs comprise 11 subfamilies and numerous spliced transcripts. Various non-specific PDE inhibitors, including propentofylline, pentoxifylline and inbudilast, have been reported to attenuate hypersensitivity in numerous animal models of neuropathic pain (13-15). Furthermore, it has been suggested that the PDE4 family may be the prevailing target of these PDE inhibitors. 
PDE4 comprises four subtypes: A, B, C and D; however, it remains to be elucidated which subtypes are the major targets until now.

One method that may be used to determine which subtype is associated with neuropathic pain is RNA interference (RNAi), which uses double-stranded RNA to effectively and specifically suppress gene expression and induce the degradation of target mRNA (16). Since the effects of this technique are transient, silencing a particular gene for $48 \mathrm{~h}$ in rat tissues offers the potential to map out the dynamic time course of the expression of various subtypes (17).

In the present study, RNAi was used to specifically silence various subtypes of the PDE4 gene, and to observe its effects on mechanical and thermal hyperalgesia, and glia-mediated spinal inflammation in rats with L5 spinal nerve ligation (SNL). The results of the present study may contribute to understanding regarding which subtype of PDE4 is associated with glial activation, and shed light on the development of novel therapeutic strategies for the treatment of neuropathic pain.

\section{Materials and methods}

Experimental animals. A total of 286 adult male Sprague-Dawley (SD) rats (weight, 180-200 g; age, 8-12 weeks) were obtained from the Experimental Animal Center of Jinling Hospital (Nanjing, China). The rats were maintained at $24 \pm 1^{\circ} \mathrm{C}$, under a $12 \mathrm{~h}$ light-dark cycle, with ad libitum access to food and water. The present study followed the International Association for the Study of Pain guidelines for pain research in animals (18), and all animal studies were approved by the Animal Care and Use Committee of Jinling Hospital.

Detection of PDE4A, B, C and D protein expression using western blotting. A total of $16 \mathrm{SD}$ rats were randomly divided into four groups ( $\mathrm{n}=4$ /group), to determine the levels of PDE4A, B, C and D in the lumbar spinal cord. The rats were sacrificed under anesthesia with sodium pentobarbital (40 mg/kg, i.p.; Suolaibao Biotechnology Co,. Ltd., Beijing, China) and lumbar spinal cords (L4 and L5) were harvested and stored at $-80^{\circ} \mathrm{C}$. Frozen tissues were homogenized in radioimmunoprecipitation assay lysis buffer containing $1 \mathrm{mM}$ phenylmethylsulfonyl fluoride (Beyotime Institute of Biotechnology, Haimen, China) and centrifuged at $4^{\circ} \mathrm{C}$ for $10 \mathrm{~min}$ at $10,000 \mathrm{x} \mathrm{g}$. The supernatants were harvested and protein concentrations were determined using the Bradford method (Leagene Biotechnology Company, Beijing, China). Protein samples $(100 \mu \mathrm{g})$ were separated by $10 \%$ sodium dodecyl sulfate-polyacrylamide gel electrophoresis and were transferred to $0.45-\mu \mathrm{m}$ polyvinylidene difluoride membranes (EMD Millipore, Billerica, MA, USA). The membranes were blocked with $5 \%$ nonfat dry milk, and were then incubated with the following rabbit anti-rat polyclonal antibodies: Anti-PDE4A (1:1,000; cat. no. ab14607), anti-PDE4B (1:1,000; cat. no. ab14611), anti-PDE4D (1:500; cat. no. ab14613) (Abcam, Cambridge, MA, USA) and anti-PDE4C (1:300; cat. no. PD4C-301AP: Fabgennix International Inc., Frisco, TX, USA) overnight at $4^{\circ} \mathrm{C}$. Subsequently, the membranes were incubated with a horseradish peroxidase (HRP)-labeled goat anti-rabbit immunoglobulin (Ig)G antibody (1:1,000; cat. no. 7074; Cell Signaling Technology, Inc., Danvers, MA, USA) for $1 \mathrm{~h}$ at room temperature. Glyceraldehyde-3-phosphate dehydrogenase (GAPDH) was used as a loading control $(1: 1,000$; cat. no. 3683; Cell Signaling Technology, Inc.). The proteins were visualized using chemiluminescence reagents provided within an enhanced chemiluminescence kit (GE Healthcare Life Sciences, Chalfont, UK) and were exposed to film. Scanning densitometry (ImageJ2x; Rawak Software, Inc.; National Institutes of Health, Bethesda, MD, USA) was used for the semi-quantitative analysis of the data.

Small interfering (si)RNA preparation. siRNA were designed to target the sequences of rat PDE4A (GenBank accession NM_013101), PDE4B (GenBank accession NM_017031) and PDE4D (GenBank accession NM_017032). The BLOCK-iT $^{\mathrm{TM}}$ Alexa Fluor Red Oligo (Invitrogen; Thermo Fisher Scientific, Inc., Waltham, MA, USA) was used as a mismatch control. All of the oligonucleotide sequences were examined against the GenBank database using the Basic Local Alignment Search Tool algorithm (http://blast.ncbi.nlm.nih. gov/Blast.cgi), in order to exclude non-specific matches with any unintended nucleotide sequences. The oligonucleotides, which had been synthesized, individually deprotected, and purified using RNase-free high-performance liquid chromatography, were purchased from Invitrogen; Thermo Fisher Scientific, Inc. The siRNA stocks were aliquoted and stored at $-20^{\circ} \mathrm{C}$, at a concentration of $200 \mu \mathrm{M}$ in annealing buffer (19). siRNA sequences used in the present study are presented in Table I.

Intrathecal catheter implantation. Under sodium pentobarbital (40 mg/kg, i.p.) anesthesia, an intrathecal catheter (PE-10 tubing, $18 \mathrm{~cm}$ ) was inserted $1-2 \mathrm{~cm}$ cephalad into the rat lumbar subarachnoid space at the L4-L5 intervertebral discs. The catheter implantation procedure was successfully performed in 224 of the 270 remaining rats. The tip of the catheter was placed near the lumbar enlargement of the spinal cord (20). The catheter, with $\sim 20 \mu 1$ dead space, was subcutaneously tunneled and externalized through the skin of the neck region. After 5 days, $2 \%$ lidocaine (10 $\mu$; Jinling Pharmaceutical Co., Ltd., Nanjing, China) was injected intrathecally into the rats with no impaired movement. Rats that exhibited lower limb paralysis within 1 min indicated successful catheterization, and those that exhibited neurological deficits resulting from the surgical procedure were excluded from further experiments.

Surgery and siRNA administration. The L5 spinal nerve ligation model was generated according to methods described by Chung et al (21). Briefly, following anesthetization with sodium pentobarbital $(40 \mathrm{mg} / \mathrm{kg}$, i.p.), the rats with intrathecal catheters were placed in the prone position. The left paraspinal muscles were separated from the spinous processes at the L4-S2 level under aseptic conditions. The L5 transverse process was carefully removed, in order to identify the spinal nerves, and the L5 spinal nerve was ligated with 7-0 silk thread. In the sham group, the surgical procedure was identical, except that the left L5 spinal nerve was not 
ligated. The rats were returned to their cages and observed for any signs of motor deficits. None of the rats exhibited motor dysfunction after surgery.

The rats were divided into seven groups ( $\mathrm{n}=32 /$ group): The sham group (sham surgery + saline), the saline group $\left(\mathrm{SNL}+\right.$ saline), the vehicle group (SNL + Lipofectamine ${ }^{\circledR}$ RNAiMAX; Invitrogen; Thermo Fisher Scientific, Inc.), the mismatch siRNA group (SNL + mismatch siRNA), the PDE4A-siRNA group (SNL + PDE4A-siRNA), the PDE4B-siRNA group (SNL + PDE4B-siRNA) and the PDE4D-siRNA group (SNL + PDE4D-siRNA). siRNA or mismatch RNA complexes were prepared immediately prior to administration by mixing the RNA solution with Lipofectamine ${ }^{\circledR}$ RNAiMAX transfection reagent, at a ratio of $1: 4(\mathrm{w}: \mathrm{v})$. The final concentration of siRNA was $2 \mu \mathrm{g}$ in $10 \mu$. siRNAs, mismatch RNA, saline or Lipofectamine ${ }^{\circledR}$ RNAiMAX were administered intrathecally just after ligation, and at 1,3,5 and 7 days after surgery.

Evaluation of thermal and mechanical hyperalgesia. Thermal and mechanical hyperalgesia were measured 1 day prior to and 2, 4, 6 and 8 days after surgery. Behavioral studies were carried out in a quiet, temperature-controlled $\left(24^{\circ} \mathrm{C}\right)$ room between the hours of 8:00 AM and 10:00 AM.

Mechanical withdrawal threshold (MWT) was measured using an Electro Von Frey anesthesiometer (Model 2390CE; IITC, Inc., Woodland Hills, CA, USA). Briefly, the rats were individually placed beneath an inverted ventilated Plexiglas cage with a metal-mesh floor, allowing access to the plantar surface of the hind paw. After $30 \mathrm{~min}$ of acclimation, gentle incremental pressure (maximum $200 \mathrm{~g}$ ) was applied using a rigid von Frey hair to the plantar surface of the ipsilateral hind paw, until the paw was withdrawn. Five tests were conducted at intervals of $5 \mathrm{~min}$ and the force $(\mathrm{g})$ applied was recorded.

Thermal withdrawal latency (TWL) was determined using radiant heat (Model 390; IITC, Inc.). Following acclimation to the Plexiglas cage $(23 \times 18 \times 13 \mathrm{~cm} ; 3$-mm-thick glass floor), the radiant heat source beneath the glass floor was focused on the plantar surface of the ipsilateral hind paw when in contact with the floor. The paw TWL was obtained five times per animal with intervals of $5 \mathrm{~min}$. Light intensity was preset, in order to obtain a baseline latency of $\sim 10 \mathrm{sec}$ and the cutoff time was set at $20 \mathrm{sec}$ to avoid tissue damage.

Detection of PDE4A, $B$ and $D$ mRNA expression. Following behavioral testing at 2, 4, 6 and 8 days after the operation, all of the rats were anesthetized with sodium pentobarbital ( $80 \mathrm{mg} / \mathrm{kg}$, i.p.) and were sacrificed by decapitation, and all appliances were treated with diethylpyrocarbonate to prevent the degradation of mRNA. The lumbar spinal cords were harvested and the mRNA expression levels of PDE4A, B and $\mathrm{D}$ were assessed using reverse transcription-quantitative polymerase chain reaction (RT-qPCR) $(n=6$ at each time point for each group). The protein expression levels of PDE4A, B and D were assessed by western blotting, as previously described.

Following homogenization of the tissue samples, total RNA was extracted from the ipsilateral lumbar spinal cord using an RNA Isolation kit (Invitrogen; Thermo Fisher Scientific, Inc.)
Table I. siRNA sequences.

\begin{tabular}{ll} 
siRNA & \multicolumn{1}{c}{ Sequence } \\
\hline siR-A & S: 5'-AAGAGUGAGAAGUUGCUUCGAACGC-3' \\
& A: 5'-UUCUCACUCUUCAACGAAGCUUGCG-3' \\
siR-B & S: 5'-UUCACCAUCCACAACAACAGUCUUG-3' \\
& A: 5'-AAGUGGUAGGUGUUGUUGUCAGAA-3' \\
siR-D & S: 5'-AUGGAUGGUUGGUUGCACAUGGGUG-3' \\
& A: 5'-CACCCAUGUGCAACCAACCAUCCAU-3'
\end{tabular}

siRNA/siR, small interfering RNA; S, sense; A, antisense.

Table II. Primer sequence used in RT-PCR.

\begin{tabular}{ll}
\hline Gene & \multicolumn{2}{c}{ Sequence } \\
\hline PDE4A & F: 5'-GAAGACAACCGGGACTCCT-3' \\
& R: 5'-CCTCAGTGGTAGGCAATCC-3' \\
PDE4B & F: 5'-CCTCCGACACCTTCGTAAC-3' \\
& R: 5'-CCAGGTCTGTGAAGACAGC-3' \\
PDE4D & F: 5'-CCCTCTTGACTGTTATCATGCACACC-3' \\
& R: 5'-GATCCTACATCATGTATTGCACTGGC-3' \\
GAPDH & F: 5'-CCATGTTCGTCATGGGTGTGAACCA-3' \\
& R: 5'-GCCAGTAGAGGCAGGGATGATGTTC-5' \\
\hline
\end{tabular}

PDE4, phosphodiesterase 4; GAPDH, glyceraldehyde 3-phosphate dehydrogenase; F, forward; $\mathrm{R}$, reverse.

and RNA was detected using a spectrophotometer (Epoch 2; BioTek Instruments, Inc., Winooski, VT, USA). cDNA was synthesized by reverse transcription (RT). The RT reaction was carried out in a $20 \mu \mathrm{l}$ total reaction volume, containing $4 \mu \mathrm{l}$ 5X RT buffer, $4 \mu 12.5 \mathrm{mM}$ dNTPs, $1 \mu 1$ Multiscribe reverse transcriptase (50 U/ $\mu \mathrm{l}$ ) (Promega Corporation, Madison, WI, USA), $1 \mu 1$ RNase inhibitor, $5 \mu 1$ RNase-free water, and $3 \mu \mathrm{g}$ DNase-treated total RNA in a $5 \mu 1$ volume. The RT reaction was carried out at $25^{\circ} \mathrm{C}$ for $10 \mathrm{~min}, 37^{\circ} \mathrm{C}$ for $120 \mathrm{~min}$, and $95^{\circ} \mathrm{C}$ for $5 \mathrm{~min}$. qPCR was conducted using a Rotor-Gene 3000 Real Time PCR system (Qiagen, Inc., Valencia, CA, USA). The sequences of the primers (Invitrogen; Thermo Fisher Scientific, Inc.) used in the present study are presented in Table II. qPCR was performed using SYBR Green I (1:20,000; Qiagen, Inc., Valencia, CA, USA), with the following cycling conditions: 1 cycle at $95^{\circ} \mathrm{C}$ for $3 \mathrm{~min}$, followed by 40 cycles at $95^{\circ} \mathrm{C}$ for $45 \mathrm{sec}, 61^{\circ} \mathrm{C}$ for $45 \mathrm{sec}, 72^{\circ} \mathrm{C}$ for $40 \mathrm{sec}$ and $80^{\circ} \mathrm{C}$ for $5 \mathrm{sec}$. The PCR reaction volume $(25 \mu \mathrm{l})$ consisted of 3 units Platinum Taq DNA polymerase, $1.5 \mathrm{mM} \mathrm{MgCl}_{2}, 205 \mu \mathrm{M}$ dGTP, dCTP, dATP and dTTP, $400 \mathrm{nM}$ forward and reverse primers, $2.5 \mu 1$ 10X PCR buffer (all Promega Corporation), $10 \mu \mathrm{l}$ SYBR Green I and $1 \mu \mathrm{l}$ cDNA. The mRNA expression levels were calculated according to relative standard curves. The curves were generated by plotting the quantification cycle $(\mathrm{Cq})$ against the log amount of total cDNA added to the reaction. The relative target gene expression levels were 
determined using the $2^{-\Delta \Delta \mathrm{Cq}}$ method (22). Results were normalized to GAPDH.

Detection of extracellular signal-regulated kinases (ERK), phosphorylated (p)-ERK, CDI1 and glial fibrillary acidic protein $(G F A P)$. A total of 8 days after surgery, the protein expression levels of ERK, p-ERK, CD11 and GFAP were detected by western blotting $(n=4)$, as described previously in the present study. For ERK and p-ERK, $50 \mu \mathrm{g}$ samples were separated in each lane. The following antibodies were used: Anti-ERK1/2 (1:1,000; cat. no. 9102), anti-p-ERK1/2 (Thr202/Tyr204) (1:1,000; cat. no. 9101) (Cell Signaling Technology, Inc.), anti-GFAP (1:500; cat. no. sc-9065; Santa Cruz Biotechnology, Inc., Dallas, TX, USA), anti-CD11b (1:1,000; cat. no. ab75476) and HRP-conjugated goat anti-mouse (cat. no. ab47827)/anti-rabbit (cat. no. ab6721) Ig $(1: 1,000)$ (Abcam).

Detection of TNF- $\alpha, I L-1 \beta, I L-6$ and $I L-10$ expression. The expression of cytokines, including TNF- $\alpha$, IL-1 $\beta$, IL- 6 and IL-10, was determined using enzyme-linked immunosorbent assay (ELISA) kits (R\&D Systems, Minneapolis, MN, USA) 8 days after surgery ( $n=4 /$ group), according to the manufacturer's protocol. Ipsilateral lumbar spinal cord samples (40-50 mg) were dissected and homogenized in a buffer containing a protease inhibitor (Roche Diagnostics, Mannheim, Germany) using a Power Gen 124 tissue tearer (Thermo Fisher Scientific, Inc.). The samples were then centrifuged at 20,000 x g for $30 \mathrm{~min}$ at $4^{\circ} \mathrm{C}$. The supernatants were aliquoted and stored at $-80^{\circ} \mathrm{C}$ for further protein quantification.

Statistical analysis. Data are presented as the mean \pm standard deviation. Data from the western blotting and RT-qPCR studies were analyzed using one-way analysis of variance (ANOVA). Data from the thermal and mechanical hyperalgesia tests were analyzed using two-way ANOVA, in order to determine the differences between the groups at various time points. All statistical analyses were performed using SPSS 18.0 statistical software (SPSS, Inc., Chicago, IL, USA). P<0.05 was considered to indicate a statistically significant difference.

\section{Results}

Protein expression levels of PDE4A, B and D in the spinal cord of naïve rats. PDE4A, B and D protein expression was detected in the spinal cord of naïve rats (Fig. 1); however, PDE4C expression was not detected (data not shown).

siRNA attenuates the protein and $m R N A$ expression levels of $P D E 4 A, B$ and D. PDE4A, B and D mRNA expression levels were significantly upregulated 2, 4, 6 and 8 days after L5 SNL, as compared with the sham group (Fig. 2). Treatment with saline, vehicle or mismatched RNA did not reduce the upregulated mRNA expression levels $(\mathrm{P}<0.05$; Fig. 2). However, treatment with $2 \mu \mathrm{g}$ siRNA-PDE4A, B or D significantly decreased the mRNA expression levels ofPDE4A, B or $\mathrm{D}$, respectively $(\mathrm{P}<0.05$; Fig. 2$)$, as compared with the saline group. This effect, first observed at 2 days post-L5 SNL, continued to 8 days post-ligation without significant alterations.
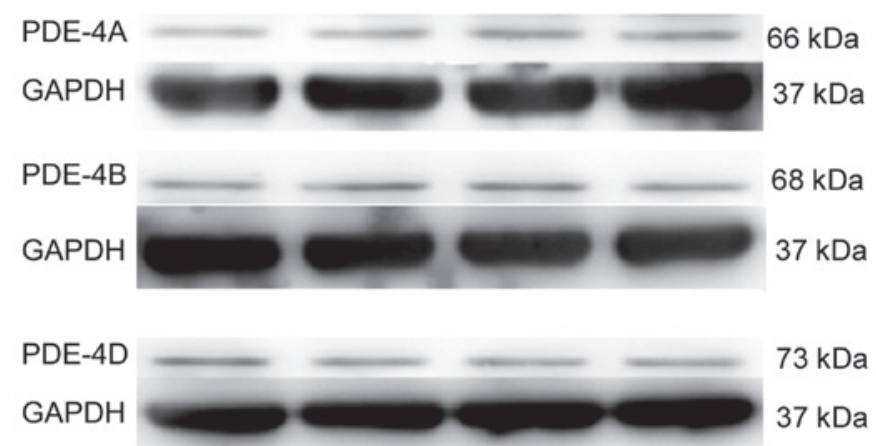

Figure 1. Protein expression of phosphodiesterase (PDE)4A, B and D in the spinal cord of naïve rats. GAPDH, glyceraldehyde 3-phosphate dehydrogenase.

siRNA exhibited similar effects on the protein expression levels of PDE4A, B and D (Fig. 3). L5 ligation resulted in overexpression of PDE proteins, and intrathecal injection with siRNA-PDE4A, B or D significantly lowered the expression of PDE4A, B or D, respectively.

Intrathecal injection with PDE4B siRNA improves MWT and TWL in SNL rats. Prior to L5 SNL, all groups exhibited comparable baseline thresholds for mechanical and thermal stimuli (Fig. 4A and B; -1 day). The values of the sham group rats were steady during the experimental period (Fig. 4A and B). L5 SNL led to significantly reduced MWT and TWL $(\mathrm{P}<0.05$; Fig. 4). No significant differences were observed between the saline, vehicle and mismatched RNA groups ( $\mathrm{P}>0.05$; Fig. 4). In addition, intrathecal injection with PDE4A-siRNA and PDE4D-siRNA did not improve the mechanical allodynia and thermal hyperalgesia, as compared with the saline group (P>0.05; Fig. 4). However, injection with PDE4B-siRNA significantly attenuated the development of mechanical allodynia and thermal hyperalgesia, as compared with the saline group $(\mathrm{P}<0.05$; Fig. 4$)$.

ERK activation in the spinal cord. L5 SNL resulted in a significant increase in the expression levels of p-ERK, as compared with the sham group (Fig. 5A; P<0.05). Compared with the saline group, only the PDE4B-siRNA group exhibited a significant decrease in the expression levels of $\mathrm{p}$-ERK (Fig. 5A; $\mathrm{P}<0.05$ ). However, there was no difference in the expression of total ERK between the various groups (Fig. 5B; $\mathrm{P}>0.05$ ).

Protein expression of $C D 11 b$ and GFAP in the spinal cord of rats. CD11b, a microglial marker (Fig. 6A) and GFAP, an astrocyte marker (Fig. 6B), were measured 8 days after L5 SNL. CD11b and GFAP protein expression levels were higher following SNL, as compared with the sham group $(\mathrm{P}<0.05$; Fig. 6). However, intrathecal injection of PDE4B-siRNA attenuated the upregulated expression of CD11b and GFAP, as compared with the saline group $(\mathrm{P}<0.05$; Fig. 6$)$.

Effects of PDE4B-siRNA on the expression of TNF- $\alpha, I L-6$, $I L-1 \beta$ and $I L-10$ in the spinal cord. To investigate the effects of PDE4B-siRNA, the expression levels of numerous inflammatory cytokines, including TNF- $\alpha$, IL-6, IL-1 $\beta$ and IL-10, 

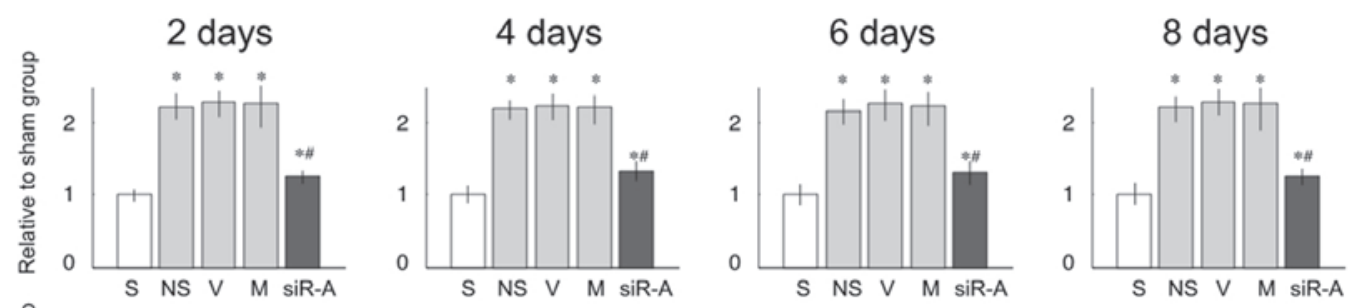

PDE4A
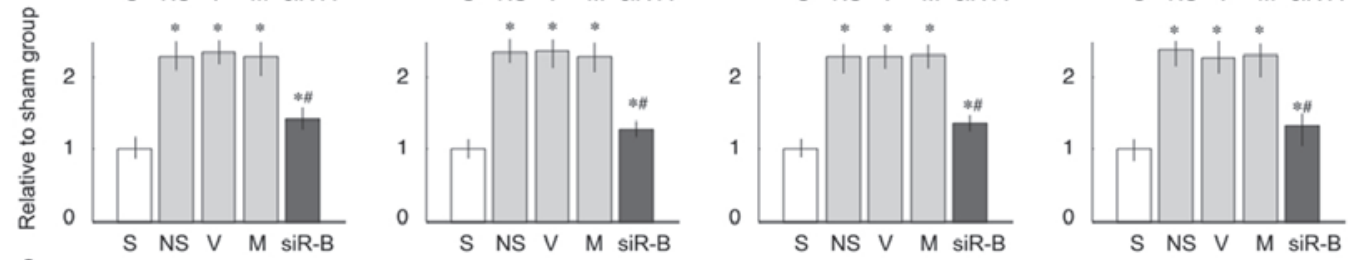

PDE4B
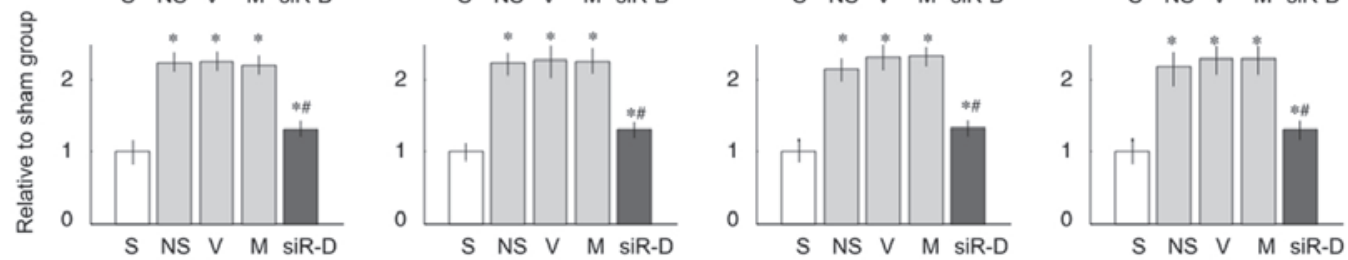

PDE4D

Figure 2. mRNA expression levels of phosphodiesterase (PDE)4A, B and D in the lumbar spinal cord of rats. Relative mRNA expression levels of PDE4A, B and D were significantly increased on days 2, 4, 6 and 8 after L5 ligation, as compared with the sham (S) group. Compared with the saline (NS) group, treatment with small interfering (si)RNA decreased the mRNA expression levels. Data are presented as the mean \pm standard deviation. ${ }^{*} \mathrm{P}<0.05$ vs. the $\mathrm{S}$ group; ${ }^{*} \mathrm{P}<0.05$ vs. the NS group. V, vehicle group; M, mismatch siRNA group; siR-A, PDE4A-siRNA group; siR-B, PDE4B-siRNA group; siR-D, PDE4D-siRNA group.

2 days
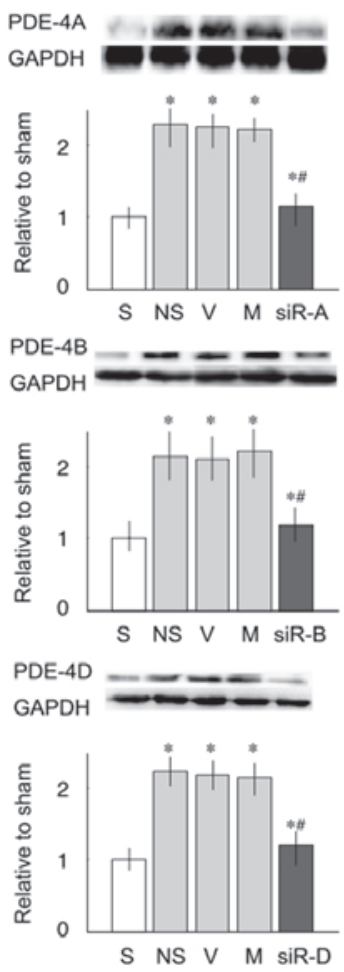

4 days
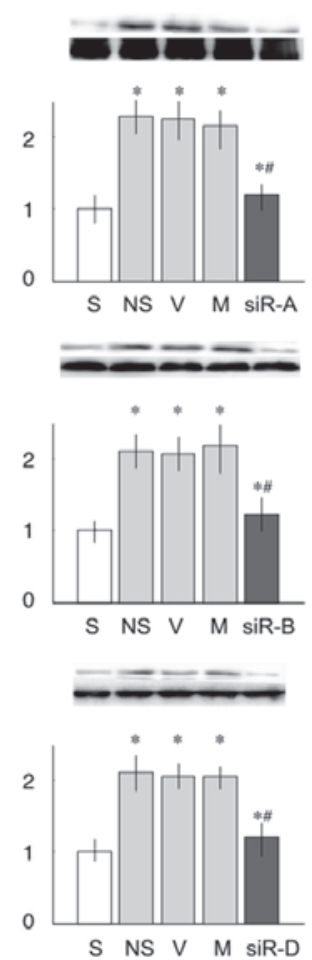

6 days
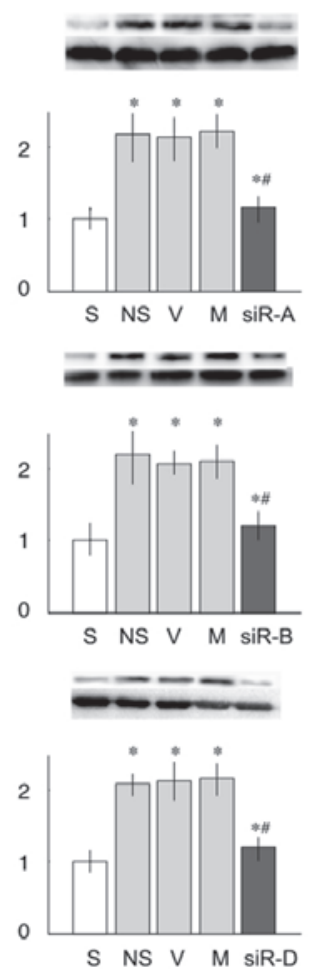

8 days
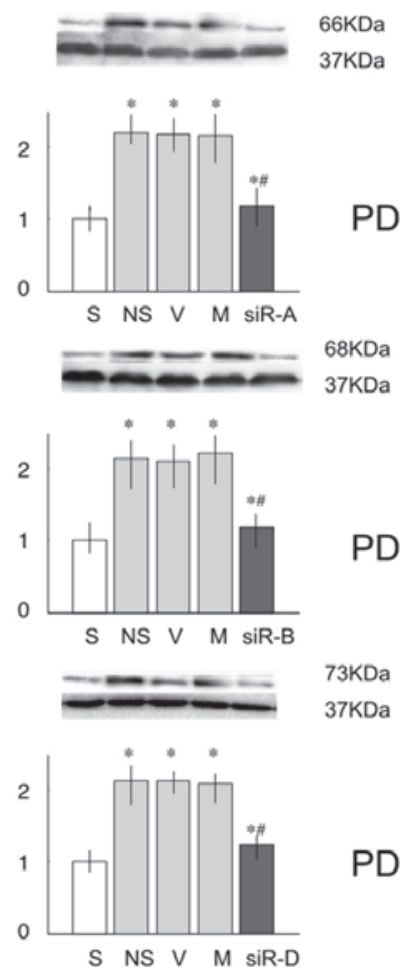

PDE4B

PDE4A

$37 \mathrm{KDa}$

PDE4D

Figure 3. Protein expression levels of phosphodiesterase (PDE)4A, B and D in the lumbar spinal cord of rats. Relative protein expression levels of PDE4A, $\mathrm{B}$ and $\mathrm{C}$ were significantly increased on days 2, 4, 6 and 8 after nerve injury, as compared with the sham (S) group. Compared with the saline (NS) group, treatment with small interfering (si)RNA decreased the protein expression levels. Data are presented as the mean \pm standard deviation. ${ }^{*}<0.05$ vs. the $\mathrm{S}$ group; ${ }^{\#} \mathrm{P}<0.05$ vs. the NS group. V, vehicle group; M, mismatch siRNA group; siR-A, PDE4A-siRNA group; siR-B, PDE4B-siRNA group; siR-D, PDE4D-siRNA group; GAPDH, glyceraldehyde 3-phosphate dehydrogenase.

were measured. Compared with the sham group, the expression levels of TNF- $\alpha$, IL- 6 and IL- $1 \beta$ were significantly increased in the SNL groups $(\mathrm{P}<0.05)$. However, the PDE4B-siRNA group exhibited significantly reduced levels 
A

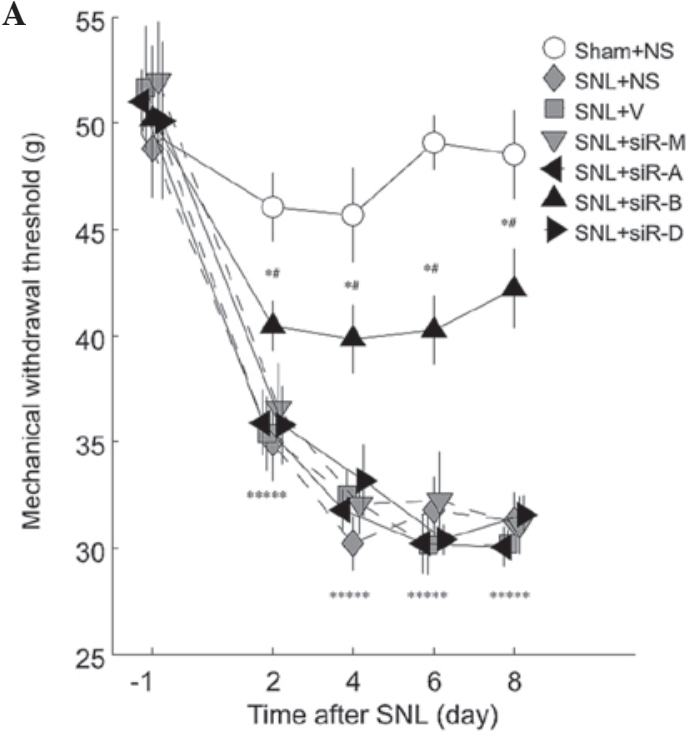

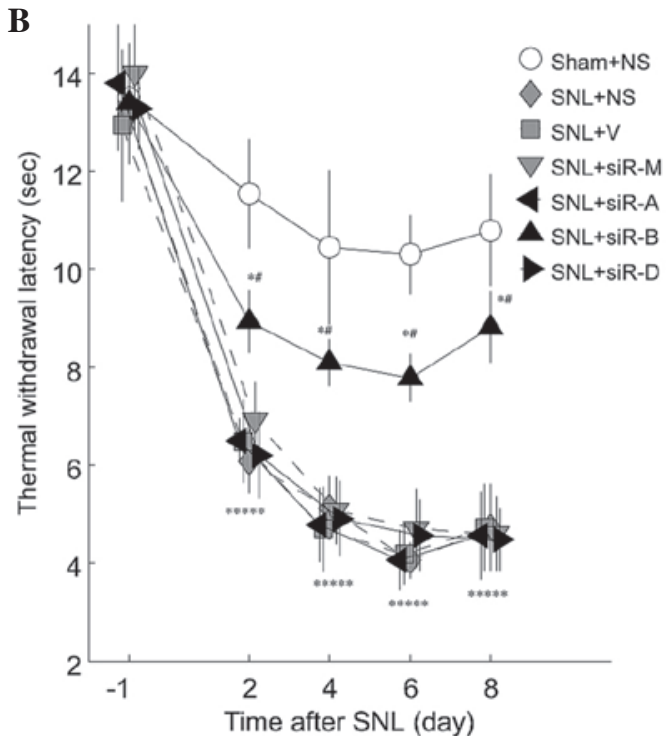

Figure 4. Line plots illustrating alterations in (A) mechanical withdrawal threshold (MWT) and (B) thermal withdrawal latency (TWL). Tests were conducted 1 day prior to surgery, and 2, 4, 6 and 8 days after the operation. L5 spinal nerve ligation (SNL) resulted in an overall significant decrease in mechanical and thermal threshold, as compared with the sham group $(\mathrm{P}<0.05)$. Treatment with PDE4-A or D-specific small interfering (si)RNA, had no effect on MWT or TWL ( $\mathrm{P}>0.05$ ), whereas PDE4B-siRNA significantly attenuated the development of mechanical and thermal hyperalgesia in L5 SNL rats, as compared with the SNL + saline (NS) group. Data are presented as the mean \pm standard deviation. ${ }^{*} \mathrm{P}<0.05$ vs. the sham group; ${ }^{*} \mathrm{P}<0.05$ vs. the $\mathrm{SNL}+\mathrm{NS}$ group. V, vehicle group; M, mismatch siRNA group; siR-A, PDE4A-siRNA group; siR-B, PDE4B-siRNA group; siR-D, PDE4D-siRNA group.

A

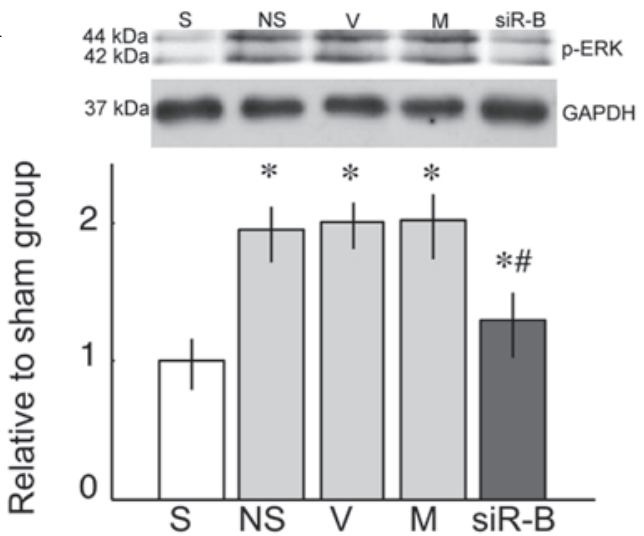

B

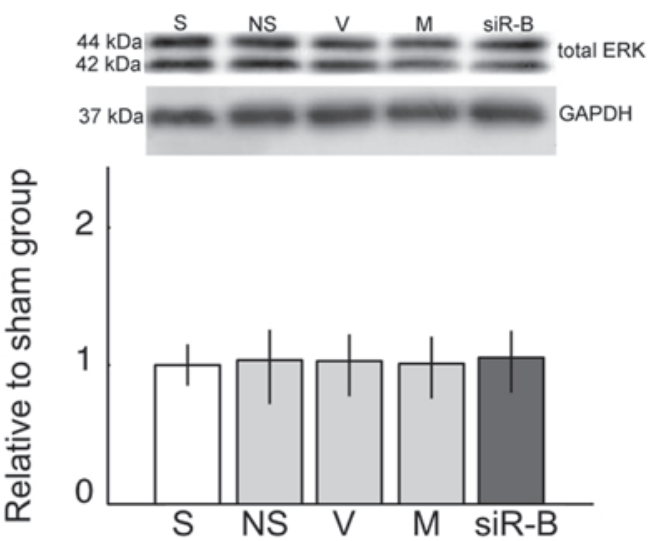

Figure 5. (A) Phosphorylated (p)-extracellular signal-regulated kinases (ERK) and (B) ERK activity in the spinal cord 8 days after surgery. p-ERK activity was markedly increased in the L5 spinal nerve ligation (SNL) groups. Treatment with phosphodiesterase (PDE)4B-small interfering (si)RNA (siR-B) significantly inhibited the activation of ERK, as compared with the saline (NS) group. However, total ERK was not significantly altered between the various groups. Data are presented as the mean \pm standard deviation. ${ }^{*} \mathrm{P}<0.05$ vs. the sham (S) group; ${ }^{\#} \mathrm{P}<0.05$ vs. the NS group. V, vehicle group; M, mismatch siRNA group; GAPDH, glyceraldehyde 3-phosphate dehydrogenase.

of the proinflammatory cytokines, as compared with the saline group $(\mathrm{P}<0.05)$. In addition, PDE4B-siRNA significantly enhanced IL-10 production, as compared with the other SNL groups $(\mathrm{P}<0.05$; Table III).

\section{Discussion}

In the present study, a model of neuropathic pain was established using single-sided L5 SNL (21). The model was confirmed, since MWT and TWL were reduced following SNL. In addition, the mRNA and protein expression levels of PDE4A, B and D were significantly upregulated following SNL. Intrathecal injection of the SNL rats with PDE4A, B or D-specific siRNA markedly reduced the elevated expression levels of PDE4A, B and D, respectively. However, only in the group treated with PDE4B-specific siRNA were MWT and TWL improved. Furthermore, only in rats treated with PDE4B-specific siRNA was p-ERK activity significantly decreased, the expression levels of proinflammatory cytokines TNF- $\alpha$, IL-1 $\beta$ and IL- 6 suppressed, and the expression of IL-10 increased 8 days after L5 SNL. These findings suggested that, among the PD4E family, PDE4B may have an important role in ameliorating neuropathic pain, potentially via inhibition of ERK activity.

In the past decade, it has been reported that microglia and astrocytes are activated in the spinal cord in various animal models of neuropathic pain, and have an important role in the development and maintenance of hypernociception $(23,24)$. 
A

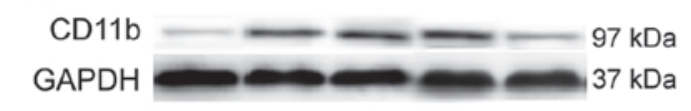

B
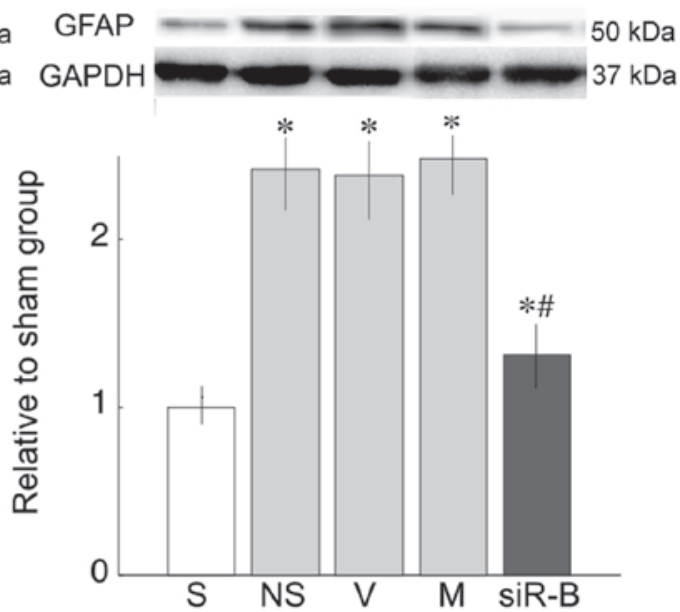

Figure 6. Protein expression levels of (A) CD11b and (B) glial fibrillary acidic protein (GFAP) in the lumbar spinal cord of rats. Relative protein expression levels of CD11b and GFAP were significantly increased 8 days after nerve injury, as compared with the sham (S) group (P<0.05). Compared with the saline (NS) group, intrathecal injection with phosphodiesterase (PDE)4B-small interfering (si)RNA (siR-B) decreased protein expression (P<0.05). Data are presented as the mean \pm standard deviation. $\mathrm{P}<0.05$ vs. the $\mathrm{S}$ group; ${ }^{\#} \mathrm{P}<0.05$ vs. the NS group. V, vehicle group; M, mismatch siRNA group; GAPDH, glyceraldehyde 3 -phosphate dehydrogenase.

Table III. Expression of cytokines 8 days post-operation ( $\mathrm{pg} / \mathrm{mg}$, mean \pm standard deviation).

\begin{tabular}{|c|c|c|c|c|c|}
\hline Cytokine & $\mathrm{S}$ & NS & V & siR-M & siR-B \\
\hline TNF- $\alpha$ & $47.6 \pm 4.3$ & $129.2 \pm 3.8^{a}$ & $126.5 \pm 4.8^{a}$ & $130.1 \pm 3.6^{\mathrm{a}}$ & $96.1 \pm 3.5^{\mathrm{a}, \mathrm{b}}$ \\
\hline IL-1 $\beta$ & $43.7 \pm 6.6$ & $144.1 \pm 15.9^{\mathrm{a}}$ & $147.6 \pm 17.6^{\mathrm{a}}$ & $154.0 \pm 11.9^{\mathrm{a}}$ & $84.2 \pm 13.3^{\mathrm{a}, \mathrm{b}}$ \\
\hline IL-6 & $77.9 \pm 12.3$ & $259.1 \pm 15.8^{a}$ & $269.4 \pm 10.3^{a}$ & $260.7 \pm 17.1^{\mathrm{a}}$ & $162.4 \pm 12.7^{\mathrm{a}, \mathrm{b}}$ \\
\hline IL-10 & $148.5 \pm 12.3$ & $156.1 \pm 9.6$ & $158.9 \pm 11.5$ & $159.8 \pm 10.9$ & $241.9 \pm 17.4^{\mathrm{b}}$ \\
\hline
\end{tabular}

TNF- $\alpha$, IL-6 and IL-1 $\beta$ expression was markedly elevated in rats following L5 ligation, as compared with the sham (S) group. Phosphodiesterase (PDE)4B-siRNA treatment significantly reduced the expression levels of TNF- $\alpha$, IL- 6 and IL-1 $\beta$, but significantly enhanced the secretion of IL-10. ${ }^{a} \mathrm{P}<0.05$ vs. the $\mathrm{S}$ group; ${ }^{\mathrm{b}} \mathrm{P}<0.05$ vs. the saline (NS) group. V, vehicle group; siR, small interfering RNA, siR-M, mismatch siR; siR-B, PDE4B siR; TNF, tumor necrosis factor; IL, interleukin.

In our previous study, a non-specific glial inhibitor, pentoxifylline, was shown to exert a dose-dependent antihyperalgesic effect when systemically injected prior to nerve injury; however, it had no effect following the establishment of hypersensitivity (14). In addition, other non-specific PDE inhibitors, such as propentofylline and inbudilast, may attenuate hyperalgesia in both preventive and therapeutic schemes; the differences may be due to the various inhibitory potencies of these drugs on PDE4 $(13,15)$. To further elucidate the role of PDE4, the present study used specific siRNA to suppress the respective PDE4 subtypes, and observed the behavioral and neuroinflammatory response in the spinal cord.

The expression levels of PDE4A, B, C and D were initially detected by western blotting in the spinal cord of naïve rats. The expression levels of PDE4A, B and D were similar and quite low, as compared with GAPDH; however, PDE4C protein was not detected. These results were consistent with those of previous reports $(25,26)$, which detected the expression of PDE4A, B and D in oligodendrocytes, and PDE4B in microglia using immunohistochemistry.
The present study used three siRNAs targeting PDE4A, $\mathrm{B}$ and $\mathrm{D}$ to study their potential roles in neuropathic pain. In order to determine the effects and specificity of the siRNA, we observed both the mRNA and protein expression levels of the respective targets 1 day after siRNA injection. After 2, 4, 6 and 8 days, SNL significantly increased the mRNA and protein expression levels of PDE4A, B and D by 200\%, and only specific siRNA could significantly inhibit this increase by $\sim 50 \%$. The possible mechanisms underlying increased PDE4 expression may be via nuclear factor- $\kappa \mathrm{B}$ activation (27) and enhanced N-methyl-D-aspartate receptor activity (28) after nerve injury. siRNA specificity was confirmed by the markedly suppressive effects of the siRNA molecules for up to 8 days after SNL.

All three siRNA were able to suppress the expression of their respective targets; however, only PDE4B siRNA exhibited significant attenuation of mechanical and thermal hyperalgesia in the SNL rats after 8 days. Previous studies reported that PDE4B is mainly expressed in microglia $(29,30)$; therefore, the expression levels of CD11b, a microglia marker and GFAP, an astrocyte marker were detected 8 days after 
SNL. The present study demonstrated that PDE4B siRNA could significantly inhibit the increases in CD11b and GFAP after nerve injury. These findings indicated that only inhibition of PDE4B could suppress the SNL-induced activation of microglia and astrocytes.

To further understand the inhibitory effects of PDE4B siRNA, alterations in ERK activity were observed in the lumbar spinal cord 8 days after SNL. Previous studies have demonstrated that phosphorylation of ERK contributes to the neuroinflammation mediated by microglia and astrocytes in various models of neuropathic pain (31-33). The present study demonstrated that PDE4B siRNA was able to significantly inhibit the activation of ERK. Although the present study did not measure the levels of cAMP in the spinal cord, previous studies have shown that an elevated cAMP signal may inhibit the activation of ERK via protein kinase A (PKA)-dependent and -independent pathways $(34,35)$.

Cytokines are important factors released by activated microglia and astrocytes, which influence neuronal excitivity. Previous studies have reported that enhanced intracellular cAMP activity in microglia and astrocytes may inhibit the production of proinflammatory cytokines and enhance IL-10 secretion $(36,37)$. Using primary cultured microglia, we further demonstrated that PKA was a main downstream effector (38). In the present study, PDE4B siRNA exerted inhibitory effects on the expression of TNF- $\alpha$, IL- $1 \beta$ and IL- 6 , and enhanced the expression of IL-10.

Considering the low expressions of PDE, $100 \mu \mathrm{g}$ protein was loaded for western blotting in the present study; however, PDE4C expression could not be detected in naïve rats. Fortunately, previous studies have demonstrated that PDE4A, $\mathrm{B}$ and $\mathrm{D}$ are expressed in oligodendrocytes, whereas only PDE4B has been detected in microglia, as determined using immunohistochemistry $(25,26)$. Secondly, the levels of cAMP were not detected in the spinal cord after intrathecal siRNA injection. Since the present study was focused on the effects of PDE on the modulation of glial-mediated neuroinflammation, it was hypothesized that the level of cAMP in the mixed spinal tissue could not reflect the levels in glia. In our ongoing study using cultured microglia, cAMP concentration was measured following treatment with PDE4 siRNA (unpublished data). Only PDE4B siRNA was able to significantly increase cAMP levels in the microglia $30 \mathrm{~min}$ after a lipopolysaccharide challenge.

The results of the present study indicated that PDE4B may be considered the main effector for the degradation of CAMP in glia, and inhibition of PDE4B may inhibit glial activation, reduce proinflammatory cytokine expression, and subsequently attenuate hypernociception following nerve injury.

In conclusion, the present study provided evidence suggesting that inhibition of PDE4B may attenuate neuroinflammation in the spinal cord, and partly relieve neuropathic pain. PDE4B may be considered a promising target for the development of novel therapeutic strategies for the treatment of neuropathic pain.

\section{Acknowledgements}

The present study was supported by the National Natural Science Foundation of China (grant no. 81102514).

\section{References}

1. Dworkin RH, O'Connor AB, Backonja M, Farrar JT, Finnerup NB, Jensen TS, Kalso EA, Loeser JD, Miaskowski C, Nurmikko TJ, et al: Pharmacologic management of neuropathic pain: Evidence-based recommendations. Pain 132: 237-251, 2007.

2. Attal N, Cruccu G, Baron R, Haanpää M, Hansson P, Jensen TS and Nurmikko T; European Federation of Neurological Societies: EFNS guidelines on the pharmacological treatment of neuropathic pain: 2010 revision. Eur J Neurol 17: e1113-e1188, 2010.

3. Gao YJ and Ji RR: Targeting astrocyte signaling for chronic pain. Neurotherapeutics 7: 482-493, 2010.

4. Tsuda M, Inoue K and Salter MW: Neuropathic pain and spinal microglia: A big problem from molecules in 'small' glia. Trends Neurosci 28: 101-107, 2005.

5. Watkins LR and Maier SF: Glia: A novel drug discovery target for clinical pain. Nat Rev Drug Discov 2: 973-985, 2003.

6. Liou JT, Liu FC, Hsin ST, Yang CY and Lui PW: Inhibition of the cyclic adenosine monophosphate pathway attenuates neuropathic pain and reduces phosphorylation of cyclic adenosine monophosphate response element-binding in the spinal cord after partial sciatic nerve ligation in rats. Anesth Analg 105: 1830-1837, 2007.

7. Taskén K and Aandahl EM: Localized effects of cAMP mediated by distinct routes of protein kinase A. Physiol Rev 84: 137-167, 2004.

8. Ottonello L, Morone MP, Dapino P and Dallegri F: Cyclic AMP-elevating agents down-regulate the oxidative burst induced by granulocyte-macrophage colony-stimulating factor (GM-CSF) in adherent neutrophils. Clin Exp Immunol 101: 502-506, 1995.

9. Pearse DD, Pereira FC, Marcillo AE, Bates ML, Berrocal YA, Filbin MT and Bunge MB: cAMP and Schwann cells promote axonal growth and functional recovery after spinal cord injury. Nat Med 10: 610-616, 2004.

10. Pryzwansky KB and Madden VJ: Type 4A cAMP-specific phosphodiesterase is stored in granules of human neutrophils and eosinophils. Cell Tissue Res 312: 301-311, 2003.

11. Lugnier C: Cyclic nucleotide phosphodiesterase (PDE) superfamily: A new target for the development of specific therapeutic agents. Pharmacol Ther 109: 366-398, 2006.

12. Lynch MJ, Hill EV and Houslay MD: Intracellular targeting of phosphodiesterase-4 underpins compartmentalized cAMP signaling. Curr Top Dev Biol 75: 225-259, 2006.

13. Gwak YS and Hulsebosch CE: Remote astrocytic and microglial activation modulates neuronal hyperexcitability and below-level neuropathic pain after spinal injury in rat. Neuroscience 161: 895-903, 2009

14. Liu J, Feng X, Yu M, Xie W, Zhao X, Li W, Guan R and Xu J: Pentoxifylline attenuates the development of hyperalgesia in a rat model of neuropathic pain. Neurosci Lett 412: 268-272, 2007.

15. Hama A, Broadhead A, Lorrain DS and Sagen J: The antinociceptive effect of the asthma drug ibudilast in rat models of peripheral and central neuropathic pain. J Neurotrauma 29: 600-610, 2012.

16. Fire A, Xu S, Montgomery MK, Kostas SA, Driver SE and Mello CC: Potent and specific genetic interference by double-stranded RNA in Caenorhabditis elegans. Nature 391: 806-811, 1998.

17. Doré-Savard L, Roussy G, Dansereau MA, Collingwood MA, Lennox KA, Rose SD, Beaudet N, Behlke MA and Sarret P: Central delivery of Dicer-substrate siRNA: A direct application for pain research. Mol Ther 16: 1331-1339, 2008.

18. Zimmerman M: Ethical guidelines for investigations of experimental pain in conscious animals. Pain 16: 109-110, 1983.

19. Elbashir SM, Harborth J, Lendeckel W, Yalcin A, Weber K and Tuschl T: Duplexes of 21-nucleotide RNAs mediate RNA interference in cultured mammalian cells. Nature 411: 494-498, 2001.

20. Kawamata T, Omote K, Kawamata M, Iwasaki H and Namiki A: Antinociceptive interaction of intrathecal alpha2-adrenergic agonists, tizanidine and clonidine, with lidocaine in rats. Anesthesiology 87: 436-448, 1997.

21. Chung JM, Kim HK and Chung K: Segmental spinal nerve ligation model of neuropathic pain. Methods Mol Med 99: 35-45, 2004.

22. Livak KJ and Schmittgen TD: Analysis of relative gene expression data using real-time quantitative PCR and the 2(-Delta Delta C(T)) Method. Methods 25: 402-408, 2001. 
23. Zhang GH, Lv MM, Wang S, Chen L, Qian NS, Tang Y, Zhang XD, Ren PC, Gao CJ, Sun XD and Lu LX: Spinal astrocytic activation is involved in a virally-induced rat model of neuropathic pain. PLoS One 6: e23059, 2011.

24. Liu PY, Lu CL, Wang CC, Lee IH, Hsieh JC, Chen CC, Lee HF, Lin HC, Chang FY and Lee SD: Spinal microglia initiate and maintain hyperalgesia in a rat model of chronic pancreatitis. Gastroenterology 142: 165-173, 2012.

25. Whitaker CM, Beaumont E, Wells MJ, Magnuson DS, Hetman M and Onifer SM: Rolipram attenuates acute oligodendrocyte death in the adult rat ventrolateral funiculus following contusive cervical spinal cord injury. Neurosci Lett 438: 200-204, 2008.

26. Schaal SM, Garg MS, Ghosh M, Lovera L, Lopez M, Patel M, Louro J, Patel S, Tuesta L, Chan WM and Pearse DD: The therapeutic profile of rolipram, PDE target and mechanism of action as a neuroprotectant following spinal cord injury. PLoS One 7: e43634, 2012.

27. Vicini E and Conti M: Characterization of an intronic promoter of a cyclic adenosine 3',5'-monophosphate (cAMP)-specific phosphodiesterase gene that confers hormone and cAMP inducibility. Mol Endocrinol 11: 839-850, 1997.

28. Hajjhussein H, Suvarna NU, Gremillion C, Chandler LJ and O'Donnell JM: Changes in NMDA receptor-induced cyclic nucleotide synthesis regulate the age-dependent increase in PDE4A expression in primary cortical cultures. Brain Res 1149 58-68, 2007.

29. Sebastiani G, Morissette C, Lagacé C, Boulé M, Ouellette MJ, McLaughlin RW, Lacombe D, Gervais F and Tremblay P: The cAMP-specific phosphodiesterase 4B mediates Abeta-induced microglial activation. Neurobiol Aging 7: 691-701, 2006.

30. Reyes-Irisarri E, Sánchez AJ, García-Merino JA and Mengod G: Selective induction of cAMP phosphodiesterase PDE4B2 expression in experimental autoimmune encephalomyelitis. J Neuropathol Exp Neurol 66: 923-931, 2007.
31. Zhuang ZY, Gerner P, Woolf CJ and Ji RR: ERK is sequentially activated in neurons, microglia, and astrocytes by spinal nerve ligation and contributes to mechanical allodynia in this neuropathic pain model. Pain 114: 149-159, 2005.

32. Choi DC, Lee JY, Lim EJ, Baik HH, Oh TH and Yune TY: Inhibition of ROS-induced p38MAPK and ERK activation in microglia by acupuncture relieves neuropathic pain after spinal cord injury in rats. Exp Neurol 236: 268-282, 2012.

33. Li W, Li Y, Zhu S, Ji Q, Shu Y, Zhang L and Liu J: Rosuvastatin attenuated the existing morphine tolerance in rats with L5 spinal nerve transection through inhibiting activation of astrocytes and phosphorylation of ERK42/44. Neurosci Lett 584:314-319, 2015.

34. Aronoff DM, Canetti C, Serezani CH,Luo M and Peters-Golden M: Cutting edge: Macrophage inhibition by cyclic AMP (cAMP): Differential roles of protein kinase A and exhange protein directly activated by cAMP-1. J Immunol 174: 595-599, 2005.

35. Emery AC and Eiden LE: Signaling through the neuropeptide GPCR PAC1, induces neuritogenesis via a single linear cAMPand ERK-dependent pathway using a novel cAMP sensor. FASEB J 26: 3199-3211, 2012.

36. Woo MS, Jang PG, Park JS, Kim WK, Joh TH and Kim HS: Selective modulation of lipopolysaccharide-stimulated cytokine expression and mitogen-activated protein kinase pathways by dibutyryl-cAMP in BV2 microglial cells. Brain Res Mol Brain Res 113: 86-96, 2003.

37. Zhao L and Brinton RD: Suppression of proinflammatory cytokines interleukin-1beta and tumor necrosis factor-alpha in astrocytes by a V1 vasopressin receptor agonist: A cAMP response element-binding protein-dependent mechanism. J Neurosci 24: 2226-2235, 2004.

38. Liu J, Zhao X, Cao J, Xue Q, Feng X, Liu X, Zhang F and Yu B: Differential roles of PKA and Epac on the production of cy tokines in the endotoxin-stimulated primary cultured microglia. $\mathrm{J} \mathrm{Mol}$ Neurosci 45: 186-193, 2011. 